\title{
Probing three-dimensional collective cancer invasion with DIGME
}

Amani A. Alobaidi and Bo Sun ${ }^{*}$

\section{*Correspondence:}

sunb@physics.oregonstate.edu Department of Physics, Oregon State University, Weniger Hall, Corvallis, OR, USA

\begin{abstract}
Background: Multicellular pattern formation plays an important role in developmental biology, cancer metastasis and wound healing. While many physical factors have been shown to regulate these multicellular processes, the role of ECM micro-to-meso scale geometry has been poorly understood in 3D collective cancer invasion.

Results: We have developed a mechanical-based strategy, Diskoid In Geometrically Micropatterned ECM (DIGME). DIGME allows easy engineering of the shape of 3D tissue organoid, the mesoscale ECM heterogeneity, and the fiber alignment of collagen-based ECM all at the same time. We have employed DIGME to study the 3D invasion of MDA-MB-231 diskoids in engineered collagen matrix. We find that the collective cancer invasion is closely regulated by the micro-to-meso scale geometry of the ECM.
\end{abstract}

Conclusions: We conclude that DIGME provides a simple yet powerful tool to probe 3D dynamics of tissue organoids in physically patterned microenvironments.

Keywords: Tumor organoid, ECM, Collective migration

\section{Background}

Invasion in three-dimensional (3D) extracellular matrix (ECM) is an important step in the lethal metastasis of tumors (Hunter et al. 2008). Although extensive studies have elucidated detailed mechanisms of single cell 3D motility (Friedl and Bröcker 2000; Grinnell and Petroll 2010; Petrie et al. 2012), and cell-ECM interactions (Bergert et al. 2012; Petrie and Yamada 2013; Doyle et al. 2015), 3D collective cancer invasion is still poorly understood (Christiansen and Rajasekaran 2006; Friedl and Gilmour 2009; Friedl et al. 2012; Carey et al. 2015). Most studies to date have focused on 2D collective cell migration. It has been shown that cell-cell adhesion (Maruthamuthu et al. 2011; Bi et al. 2015; Bazelliéres et al. 2015), exclusion volume (Angelini et al. 2011), contact inhibition (Liu et al. 2011; Zimmermann et al. 2016), cell-secreted chemical factors (Theveneau et al. 2013), and substrate-mediated mechanical forces (Ma et al. 2013) coordinate the multicellular motility and pattern formation of multiple cells in 2D. These results, however, have limited applicability in 3D tumor progression. The topological connectivity and porosity of 3D ECM allow cells to avoid touching one another while migrating, thus mechanical signaling via direct cell-cell contact is less important for collective motion in 3D than in 2D (Liu et al. 2013; Liang et al. 2016). Similarly, chemical signaling in 3D suffers from rapid dispersive dilution, thus the diffusion-mediated 3D intercellular correlations are much weaker compared to the case in 2D (Sun et al. 2012).

(c) The Author(s). 2017 Open Access This article is distributed under the terms of the Creative Commons Attribution 4.0 International License (http://creativecommons.org/licenses/by/4.0/), which permits unrestricted use, distribution, and reproduction in any medium, provided you give appropriate credit to the original author(s) and the source, provide a link to the Creative Commons license, and indicate if changes were made. 
To probe the collective cell migration and morphogenesis, 2D cell patterning and substrate engineering has provided much insights. For instance, various types of wound healing assays have been developed to explore the invasion of cancer cell colonies into extracellular voids of pre-defined geometries (Poujade et al. 2007; Brugués et al. 2014; Ravasio et al. 2015). These assays typically use soft-lithography fabricated stamps when seeding the cells, and lift the stamps after the cells have adhered to the substrate. Alternatively, geometric patterned cell adhesives and cell repellents (Singhvi et al. 1994; Rape et al. 2011; Deforet et al. 2014), as well as microfluidics channels (Huang et al. 2011) have been employed to restrict cell migration. By engineering the confining geometry of the substrate, emergent multicellular dynamics, such as spontaneous rotation in circular geometry (Notbohm et al. 2016), and directed migration in ratchet geometry (Mahmud et al. 2009) have been observed.

To probe 3D collective cancer invasion, multicellular tumor spheroid models have been widely employed (Inch et al. 1970; McCredie et al. 1971; Hirschhaeuser et al. 2010). Tumor spheroids are aggregates of cancer cells that preserve native 3D cell-cell contact, mimicking the configuration of solid tumors (Weiswald et al. 2015). Multiple methods have been developed to grow 3D tumor spheroids, such as the hanging droplets (Timmins and Nielsen 2007), non-adhesive microplates (Gong et al. 2015), and bi-phase liquid systems (Han et al. 2015). However, these techniques can neither control the geometry of the cell aggregates, nor have the capability of engineering complex extracellular environment. Other methods, such as 3D tissue printing (Villar et al. 2013) and photo-sensitive hydrogel (Hahn et al. 2006; Kloxin et al. 2009; DeForest and Anseth 2011; Applegate et al. 2015) are capable of 3D cell-ECM patterning at the price of expensive equipment, nonnative ECM composition, or sophisticated sample preparation (Yanagawa et al. 2016). As an alternative, we extend the mold-based technique developed by Nelson et al. (2006) into a low-cost, flexible strategy, Diskoid In Geometrically Micropatterned ECM (DIGME). DIGME is mechanical-based, and is compatible with a wide range of cells and ECM types. As we will demonstrate below, DIGME combines the powers of 3D tumor organoids and 3D ECM patterning, allowing us to independently control the shape of tumor organoids, microstructure and spatial heterogeneity of the ECM simultaneously .

\section{Methods}

\section{Instrumentation}

The basic setup of DIGME consists of a $x-y-z$ translational stage to hold sample dish, and a rotational motor to mount the mold above the sample stage. We have used parts from Thorlabs Inc. and TA instruments to assemble prototypes of DIGME. When necessary, we have also placed the DIGME setup on an inverted microscope (Leica Microsystems) to help with alignment and positioning the mold. See Additional file 1: Section S1 for schematic design of the DIGME setup.

\section{Preparation of the collagen gel}

High concentration collagen solution $(10 \mathrm{mg} / \mathrm{ml}$, Corning) is diluted and neutralized to desirable concentrations with cell growth medium (see cell culture), $\mathrm{NaOH}$ and 10X PBS, all purchased from Sigma-Aldrich. The neutralized, ice cold solution is first poured into the sample dish mounted on the DIGME setup where the mold is approximately $200 \mu \mathrm{m}$ above the glass bottom of the dish. After curing in the DIGME setup for 30-50 min (with 
the mold statically immersed or rotatin in the geling solution), we lift the mold out of the dish via the $\mathrm{z}$-motor of the translational stage. The collagen solution will continue the gelation process for another $40 \mathrm{~min}$. The molded gel is then immersed with fresh growth medium and stored at $4{ }^{\circ} \mathrm{C}$ for up to 2 days before adding cells.

\section{Cell culture and microscopy}

GFP-labeled MDA-MB-231 cells (Cell Biolabs Inc.) are maintained according to the vendor's protocol. After embedding the cells, DIGME devices are kept in tissue culture incubator except when taken out for imaging. For confocal imaging, we use a Leica SPE microscope. 10X oil immersion objective is used when confocal reflection imaging of collagen fiber is needed. Otherwise, $4 \mathrm{X}$ air objective is used to image the fluorescently labeled cells and fluorescent particles embedded in the collagen matrix. The z-stacks of confocal imaging are taken with $2 \mu \mathrm{m}$ z-steps. Confocal images are further processed in NIH ImageJ and MATLAB.

\section{Results}

To demonstrate the working principles and biocompatibility of DIGME, we first formed a cylindrical MDA-MB-231 tumor diskoid in 3D type-I collagen gel. Briefly, a stainless steel needle is used to mold the collagen gel (surrounding ECM) with a cylindrical well. The well is then filled with neutralized cell-collagen solution. After cells quickly sediment down to the well bottom (within $1 \mathrm{~min}$ ), collagen solution continues to polymerize and eventually forms the host ECM that covers the cell aggregate - a diskoid - on the bottom of the well (Fig. 1A). Within $24 \mathrm{~h}$ of incubation, cells start to invade the surrounding ECM. Figure 1B1-B3 demonstrate the top views ( $x-y$ plane) and side views ( $x-z$ plane) of a DIGME sample at day 1, 5 and 10. Notice that although the surrounding ECM and the host ECM are polymerized at the same temperature $\left(21^{\circ} \mathrm{C}\right)$ and have the same concentration $(1.5 \mathrm{mg} / \mathrm{ml})$, the invasion in the radial direction is much more pronounced compared with the spreading in the $\mathrm{z}$ direction. The biased migration direction is presumably a collective phenomena due to the cell-cell interactions (Liu et al. 2013; Valencia et al. 2015).

DIGME allows continuous confocal imaging at the single cell level, therefore we can track the morphological profiles of the diskoid over time. Cells in the diskoid generally exhibit two distinct morphologies: elongated cells are typically fast moving and strongly contracting, while rounded cells migrate with short persistence and exert only weak traction forces (Giri et al. 2013; Kim et al. 2016). Empirically, we distinguish elongated and rounded cells based on the cell aspect ratio with a threshold value of 2 . We have manually located the center of each cell and have classified each cell into elongated or rounded phenotypes as shown in Fig. 1C1-C3.

In order to quantify the diskoid invasion profile, we have located the invasion fronts by projecting the confocal images onto the $x-y$ plane. The invasion front can be described as $d_{f}(\theta, t)$, where $d_{f}$ measures the radial distance from the center of the well, $\theta$ is the polar angle, and $t$ is the time of diskoid invasion. Figure 2a shows the invasion fronts at days 0 , 3,5 , and 10 . After averaging over the polar angle $\theta$, Fig. $2 \mathrm{~b}$ shows the means and standard deviations of $d_{f}$. The invasion front grows sublinearly with time, which is consistent with the observation on tumor spheroid (Valencia et al. 2015).

In order to quantify the morphological evolution of the diskoid, we have calculated the elongated, rounded and full cell density using k-nearest neighbors of each cell. Briefly, for 


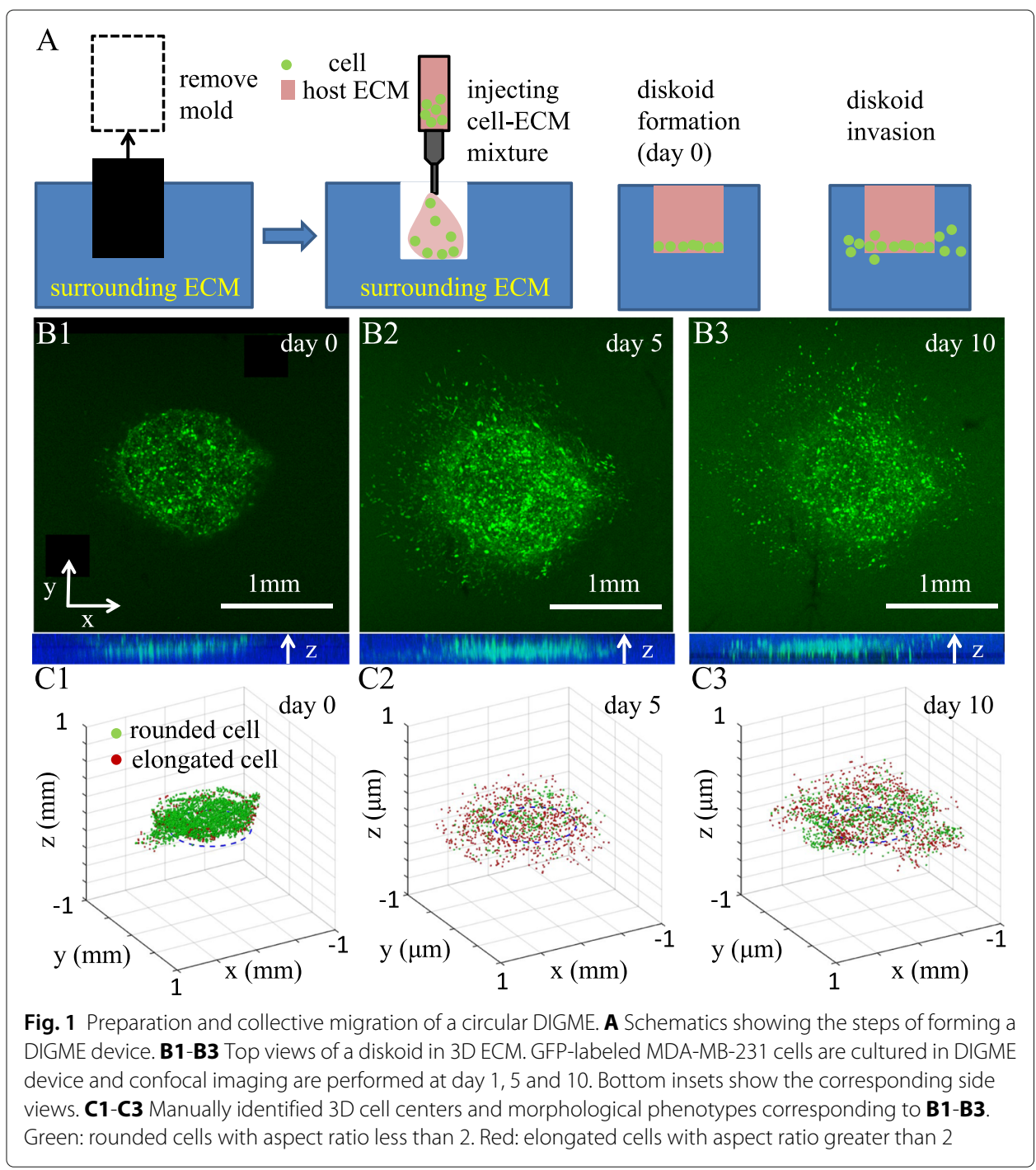

each cell $i$ at location $\mathbf{r}_{i}=\left[x_{i}, y_{i}, z_{i}\right]$, we find the minimal sphere centered at $\mathbf{r}_{i}$ with radius $r_{m}$ that encloses exactly $\mathrm{k}$ cells. The cell density at $\mathbf{r}_{i}$ is approximated to be $\rho_{d}\left(\mathbf{r}_{i}\right)=\frac{3 k}{4 \pi r_{m}^{3}}$. For simplicity, we have chosen $k=10$. Figure 2c-d show the cell density at varying invasion depth $\rho_{d}(d)$, where $d=\sqrt{x^{2}+y^{2}}$. At day 3, only a small number of cells have migrated far from the seeding radius (original diskoid-ECM interface) at $a=370 \mu \mathrm{m}$, and these cells are mostly elongated. Close to the center, cell density is approximately constant for $d \leq 300 \mu \mathrm{m}$. As invasion proceeds, the region of constant cell density expands. At the same time, cell density in this region decreases because the cell proliferation is slow compared to the migration-induced dilution. After invading the surrounding ECM for 10 days, both elongated and rounded cells are uniformly distributed for $d \leq 1 \mathrm{~mm}$, and the cell density has dropped by more than four folds. To further quantify the morphological distribution, we have normalized the invasion depth $d$ with respect to the seeding radius $a$ of the diskoid and have calculated the fraction of elongated cells $f_{\text {elongate }}$ in different regions of $d / a$. As shown in Fig. 3a, at day $3 f_{\text {elongate }}$ increases rapidly at greater radial distance, consistent with the fact that elongated cells are scout cells during collective invasion. The 

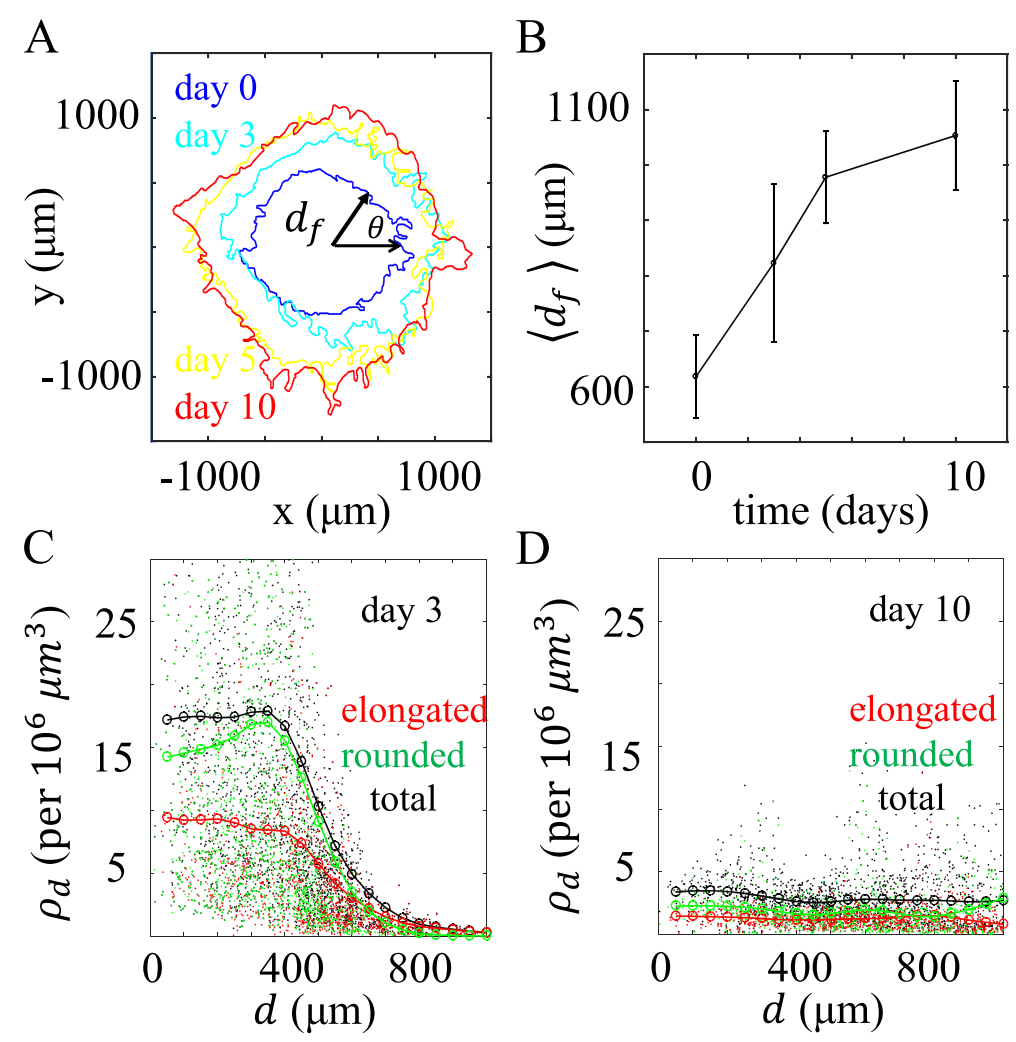

Fig. 2 The invasion profile of a DIGME device. a The invasion fronts at day 0, 3, 5, and 10 of the same sample described in Fig. 1. $\mathbf{b}$ The means and standard deviations of the invasion distance obtained by averaging $d_{f}(\theta)$ over the polar angle $\theta$ of $\mathbf{a}$. c-d Scattered plots: the local cell density by counting only the elongated cells (red), or only the rounded cells (green), or all cells (black). Lines: Running average of the scattered data points with a Gaussian kernel. c shows the results at day 3, d shows the results at day 10
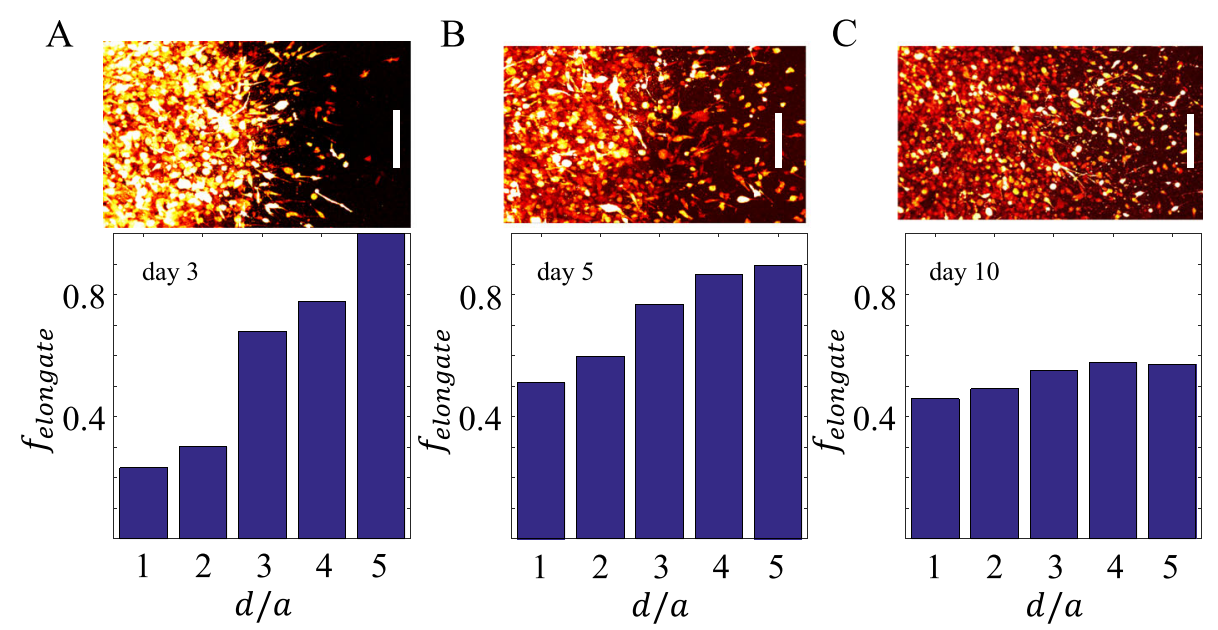

Fig. 3 The spatial-temporal profiles of cell morphology. a-c Fraction of elongated cells $f_{\text {elongate }}$ at varying distances from the center. Here the distance is normalized by the seeding radius $a=370 \mu \mathrm{m}$ of the same diskoid described in Fig. 1. Top insets: a section of the top view invading diskoid taken at days 3,5 and 10. Scale bars of the insets: $200 \mu \mathrm{m}$ 
positive correlation between $f_{\text {elongate }}$ and $d / a$ gradually diminishes over time (Fig. 3b). At day 10, a half-half mixture of elongated and rounded cells are found in all regions of the sample. To quantitatively account for these observations, we have developed a simple model based on persistent random walks. We assume the cells stochastically transform between elongated and rounded phenotypes at a rate of $\tau_{\text {trans }}$, and the two phenotypes have distinct migration persistent time $\tau_{e l}$ and $\tau_{r d}$. As elaborated in the Additional file 1, the model agrees well with the results of Figs. 2 and 3.

Solid tumors may develop various shapes in vivo, resulting in a diverse range of interfacial geometry between the cells and the ECM. DIGME allows us to easily control the geometry of diskoid. To demonstrate the capability, we employed laser-micromachining to fabricate stainless steel needles with hexagon and triangle cross-sections. Using these needles as the mold, we have generated hexagonal and triangular MDA-MB-231 diskoids in 3D collagen ECM (Fig. 4A1 and B1). We find that the original shapes of the diskoid (Fig. 4A2 and B2) largely determine the invasion pattern after 5 days of incubation (Fig. 4A3 and B3). Previously it was reported that the interfacial geometry regulates the tumorigenicity by promoting cancer-stem cells (Lee et al. 2016). Using DIGME, we show that the geometric control can be realized in truly $3 \mathrm{D}$ setups.

The extracellular space along the invasion path of a tumor is spatially heterogeneous (Shin et al. 2014). Employing DIGME, we can program the ECM heterogeneity and study its effect on the collective cancer invasion. As a proof of concept, we have formed a MDAMB-231 diskoid confined within a two-layer ECM. This is done by sequentially applying two circular molds of different diameters, and filling the coaxial wells with different concentrations of collagen gels (Fig. 5A1-A3). The inner layer, with collagen concentration $1.5 \mathrm{mg} / \mathrm{ml}$ is more porous compared to the outer layer, which has collagen at

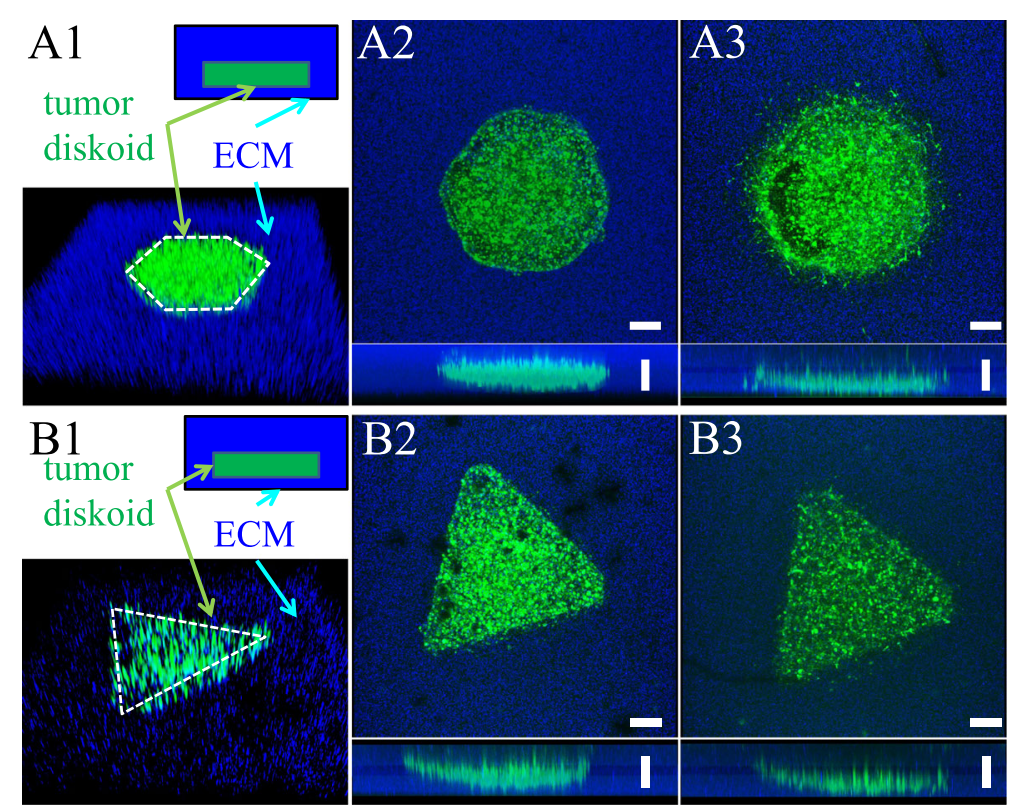

Fig. 4 The hexagonal and triangular diskoid in DIGME devices. A1 and (B1): 3D view of the diskoids. Blue: The surrounding ECM embedded with fluorescent particles. Green: GFP-labeled MDA-MB-231 cells. The seeding geometry of the diskoids are outlined in white. A2-A3 Top and side views of the hexagonal diskoid at day 0 and day 5. B2-B3, Top and side views of the triangular diskoid at day 0 and day 5. Scale bars: $200 \mu \mathrm{m}$ 

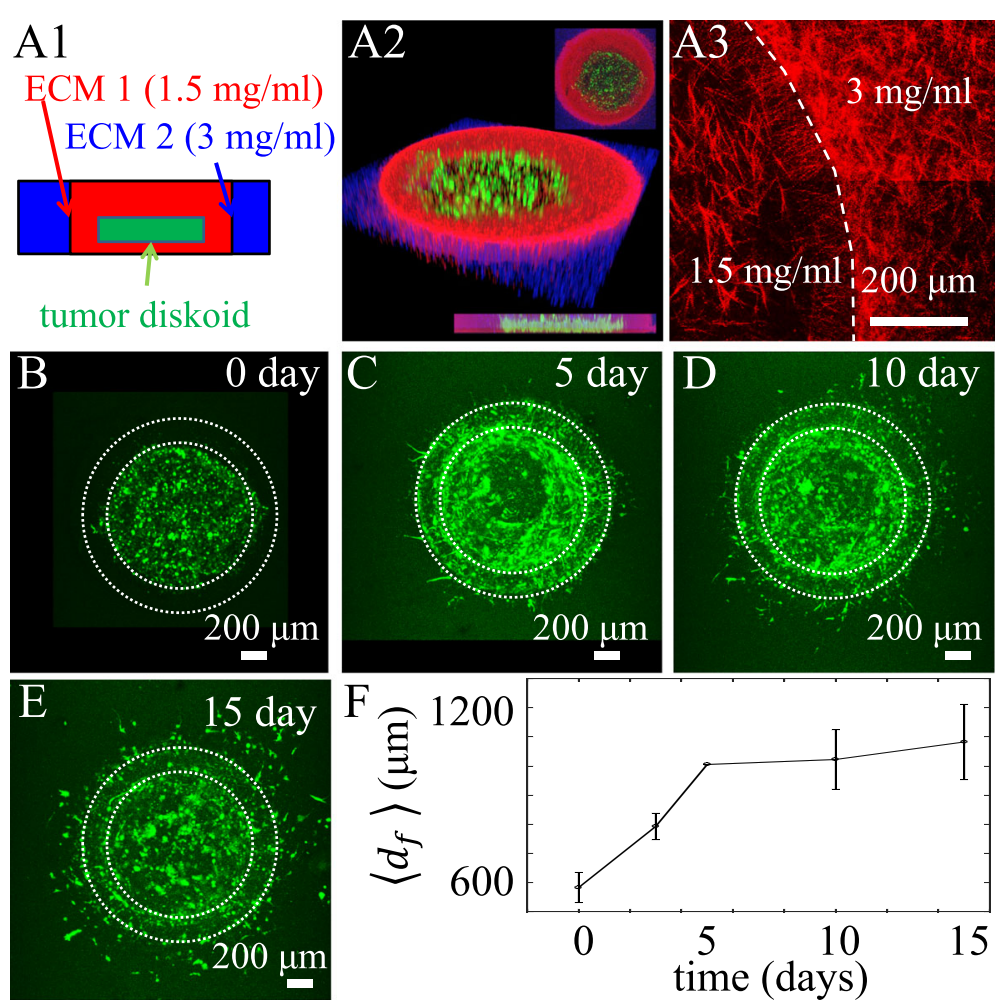

Fig. 5 A two-layer DIGME device. A1: Schematics of the two-layer device. A circular MDA-MB-231 diskoid is confined by $1.5 \mathrm{mg} / \mathrm{ml}$ collagen matrix (ECM 1). ECM 1 is inside of $3 \mathrm{mg} / \mathrm{ml}$ collagen matrix (ECM 2). A2: 3D, top, and side views of the device. Green: MDA-MB-231 cells. Red: ECM 1 labeled with red fluorescent particles, Blue: ECM 2 labeled with far-red fluorescent particles. A3: confocal reflection image showing the collagen fibers at the interface of ECM 1 and ECM 2. B-E Top views of the invading diskoid at day 0, 5, 10 and 15. $\mathbf{F}$ : Invasion distance $d_{f}$ as a function of time

concentration of $3 \mathrm{mg} / \mathrm{ml}$ (Fig. 5A3). We have observed the invasion of the diskoid for over 15 days, and the top views of the sample at days $0,5,10$ and 15 are shown in Fig. 5B-E. Within 5 days after initial seeding, the invasion front quickly reaches the interface of the inner and outer layer of the ECM (Fig. 5C). At day 5, most front cells polarize tangentially at the interface, with a few leading cells polarizing radially and starting to invade the outer ECM layer. By quantifying the invasion front profiles, we find that that invasion speed is significantly reduced at day 5 . Due to the change of cell orientation as well as the ECM microstructure, the invasion speed reduces rather abruptly at the interface between the two ECM layers. Ductal carcinoma is the most common type of breast cancer. At the early stage of ductal carcinoma, tumor cells are surrounded by a collagen matrix that are polarized long the tumor-stromal interface (Fig. 6a) (Provenzano et al. 2006). The orientation of collagen fibers becomes disorganized even perpendicularly aligned during tumor progression, correlating with the clinical outcome of cancer patients (Conklin et al. 2011). Employing DIGME, we can control the orientation of collagen fibers in the surrounding matrix, mimicking different stages of ductal carcinoma. As an example, we mount a 150 $\mu \mathrm{m}$ diameter needle approximately $300 \mu \mathrm{m}$ off the rotational axis of DIGME. While the $1.5 \mathrm{mg} / \mathrm{ml}$ collagen gel is forming, we continuously rotate the needle at $1 \mathrm{~Hz}$ for $5 \mathrm{~min}$. The microscopic flow driven by the needle aligns the collagen fibers, and the fiber orientation is subsequently locked by the gelation process (Guo and Kaufman 2007). At the 

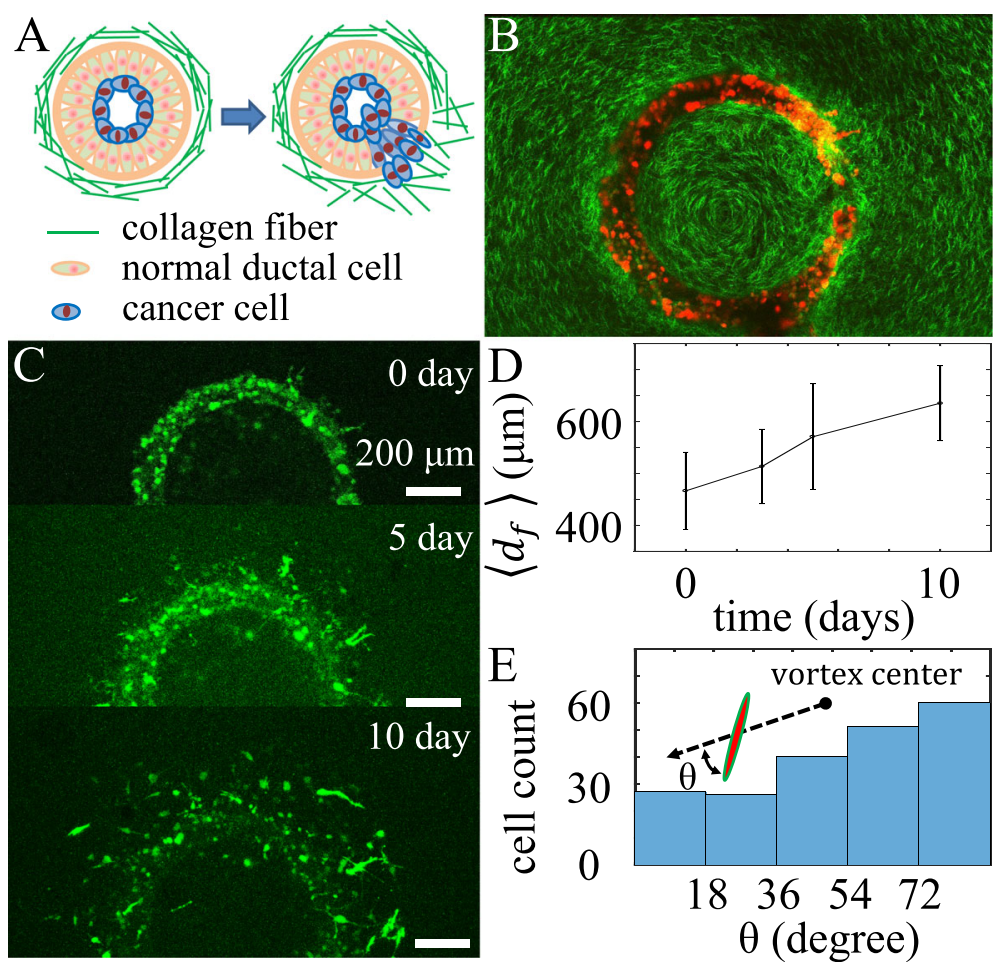

Fig. 6 A ring diskoid in circularly aligned collagen matrix simulating a ductal carcinoma. a schematics showing the invasion of a typical ductal carcinoma. b A confocal slice showing the MDA-MB-231 ring diskoid (red) surrounded by circularly polarized collagen fibers (green). c Top views of the sample at day 0,5, and 10. $\mathbf{d}$ Invasion distance $d_{f}$ as a function of time. e Histogram of cell orientation $\theta$ at 10 days. $\theta$ is the angle between the cell long axis and the local radial direction measured from the seeding center of the diskoid $C_{v}$

same time, the rotating needle carves a ring in the collagen gel, which we fill with MDAMB-231 cells mixed in the host ECM (Fig. 6b). The host ECM consists of $1.5 \mathrm{mg} / \mathrm{ml}$ collagen matrix that is randomly oriented. We have imaged the invasion process of the diskoid for 10 days, and find that the circularly polarized collagen fibers strongly impact the motility and morphology of MDA-MB-231 cells. The front invasion speed (Fig. 6d) is noticeably slower compared with the diskoid in randomly oriented matrix (Fig. 2b), and that a large fraction of cells are oriented tangentially along the collagen fibers (Fig. 6e). These results are consistent with the contact guidance observed for single cells in both 2D and 3D cultures (Kim et al. 2012).

\section{Discussion and conclusion}

We have described DIGME as a low-cost, easy-to-implement strategy to engineer the geometric microenvironment of tumor organoids. The shape-programmable organoid diskoid, preserves the native cell-cell contacts in 3D ECM, and allow us to study the single cell dissemination and cohesive progression during collective cancer invasion.

For a thin circular diskoid in isotropic homogeneous ECM, we have shown that the collective invasion and morphological evolution of MDA-MB-231 cells (Figs. 1, 2 and 3) follow the similar patterns observed in the middle plane of tumor spheroids 
(Valencia et al. 2015). Tumor spheroids are often too dense to image through directly, and requires destructive pre-imaging preparations such as cryo-section. DIGME, on the other hand, provides an alternative model allowing continuous, long-term imaging at the single cell level.

We find that the invasion profile correlates with the seeding geometry, as shown in the hexagonal and triangular diskoids (Fig. 4). It has been proposed that physical forces generated by the cellular traction propagate over the ECM, and coordinate the 3D collective cancer invasion (Wang et al. 2014; Valencia et al. 2015; Liang et al. 2016). By controlling the shape of diskoids as well as the ECM microstructure, we can tune the stress distribution in the ECM. Therefore DIGME provides an ideal experimental system to understand the mechanical mechanisms coordinating the self-organized collective cancer invasion (Gjorevski et al. 2015).

We find that 3D collective cancer invasion is regulated by the spatial heterogeneity, as well as the microscopic anisotropy of the ECM. A progressing tumor encounters dramatically varying microenvironments, or microniches (Junttila and de Sauvage 2013). By employing DIGME, we can further extend the examples demonstrated in Figs. 5 and 6 to generate complex microniches in the ECM. For instance, epithelial cells and fibroblast cells can be embedded in different layers of ECM. Also the level of ECM fiber alignment can be controlled by varying the rotational protocol that drives the DIGME mold. Such protocols, including changing the rotational speed, or implementing bidirectional rotation, can be easily realized with programmable rotary motors.

In the above, we have used breast cancer cell line MDA-MB-231 cells to demonstrate the capabilities of DIGME. It is expected that DIGME methods equally apply to any cells compatible with 3D culture, such as fibroblast cells, endothelial cells, stem cells and neural cells. Similarly, type I collagen ECM can be replaced by other forms of ECM in DIGME, including tissue-derived ECM like matrigel, and synthetic ECM like peptide gel. With these extensions, DIGME is not only useful to probe the collective invasion of tumors, but also allows one to study $3 \mathrm{D}$ multicellular dynamics in wound healing, angiogenesis, development, and tissue remodeling.

We notice that the current form of DIGME has several limitations. First of all, DIGME only control the cross-sectional shape of the diskoid, rather than the full 3D geometry of the tumor organoid. Although variants of DIGME is possible, for instance by using a cone-shaped mold, fully 3D patterning may require incorporating other techniques such as directed self-assembly (Varner and Nelson 2014). Second, metal mold fabrication has a typical tolerance of tens of micrometers, or the size of a cell. In order to control the diskoid shape down to subcellular accuracy, one may incorporate other micro-fabrication techniques, such as polymer laser micromachining or deep reactive-ion etching (Liu et al. 2011). These alternative fabrication methods have micrometer $\mu \mathrm{m}$ resolution, but may require surface treatment to ensure low-binding affinity to collagen. Third, the mechanical-based DIGME method requires one to two hours to prepare each sample. To improve the throughput, one may take advantage of the low-cost and simple operation of DIGME and implement automated parallel processing. Finally, DIGME patterns densely populated cells in $3 \mathrm{D}$, which is different from the pathological case where the tumor is grown from a few transformed cells. Future in vivo studies will be required to validated the biological insights obtained from DIGME devices, such as the invasion dynamics and drug responses. 


\section{Additional file}

Additional file 1: Supplementary Information. (PDF $1700 \mathrm{~kb}$ )

\section{Abbreviations}

DIGME: Diskoid in geometrically micropatterned ECM; ECM: Extracellular matrix

\section{Acknowledgements}

A. A. is supported by a scholarship from the Culture Mission of the Royal Embassy of Saudi Arabia (SACM). B. S. thanks Oregon State University for startup support.

\section{Availability of data and materials}

The datasets supporting the conclusions of this article are available upon request to the corresponding author. Sample datasets have been uploaded to https://figshare.com/account/home\#/projects/17375.

\section{Authors' contributions}

BS designed the research, AA performed experiments, AA and BS analyzed data and wrote the paper. Both authors read and approved the final manuscript.

\section{Ethics approval and consent to participate}

Not applicable.

\section{Consent for publication}

Not applicable.

\section{Competing interests}

The authors declare that they have no competing interests.

\section{Publisher's Note}

Springer Nature remains neutral with regard to jurisdictional claims in published maps and institutional affiliations.

Received: 23 September 2016 Accepted: 14 September 2017

Published online: 01 November 2017

\section{References}

Angelini TE, Hannezo E, Trepat X, Marquezd M, Fredberg JJ, Weitz DA. Glass-like dynamics of collective cell migration. Proc Natl Acad Sci. 2011;108(12):4714-719.

Applegate MB, Coburn J, Partlow BP, Moreau JE, Mondia JP, Marelli B, Kaplan DL, Omenetto FG. Laser-based three-dimensional multiscale micropatterning of biocompatible hydrogels for customized tissue engineering scaffolds. Proc Natl Acad Sci. 2015;112:12052.

Bazelliéres E, Conte V, Elosegui-Artola A, Serra-Picamal X, Morcillo MB, Roca-Cusachs P, Mun̈oz JJ, Sales-Pardo M, Guimerá R, Trepat X. Control of cell-cell forces and collective cell dynamics by the intercellular adhesome. Nat Cell Biol. 2015;17:409. doi:10.1038/ncb3135.

Bergert M, Chandradoss SD, Desai RA, Paluch E. Cell mechanics control rapid transitions between blebs and lamellipodia during migration. Proc Natl Acad Sci USA. 2012;109(36):14434-39.

Bi D, Lopez JH, Schwarz JM, Manning LM. A density-independent rigidity transition in biological tissues. Nat Phys. 2015;11:1074. doi:10.1038/nphys3471.

Brugués A, Anon E, Conte V, Veldhuis JH, Gupta M, Colombelli J, Mun̈oz JJ, Brodland GW, Ladoux B, Trepat X. Forces driving epithelial wound healing. Nat Phys. 2014;10:683. doi:10.1038/nphys3040.

Carey SP, Rahman A, Kraning-Rush CM, Romero B, Somasegar S, Torre OM, Williams RM, Reinhart-King CA. Comparative mechanisms of cancer cell migration through $3 \mathrm{~d}$ matrix and physiological microtracks. Am J of Physiology - Cell Physiology. 2015;308(6):436. doi:10.1152/ajpcell.00225.2014.

Christiansen JJ, Rajasekaran AK. Reassessing epithelial to mesenchymal transition as a prerequisite for carcinoma invasion and metastasis. Cancer Res. 2006;66:8319.

Conklin MW, Eickhoff JC, Riching KM, Pehlke CA, Eliceiri KW, Provenzano PP, Friedl A, Keely PJ. Aligned collagen is a prognostic signature for survival in human breast carcinoma. Am J Path. 2011;178(3):1221. doi:10.1016/j.ajpath.2010. 11.076.

DeForest CA, Anseth KS. Cytocompatible click-based hydrogels with dynamically tunable properties through orthogonal photoconjugation and photocleavage reactions. Nat Chem. 2011;3:925.

Deforet M, Hakim V, Yevick HG, Duclos G, Solberzan P. Emergence of collective modes and tri-dimensional structures from epithelial confinement. Nat Commun. 2014;5:3747.

Doyle AD, Carvajal N, Jin A, Matsumoto K, Yamada KM. Local 3d matrix microenvironment regulates cell migration through spatiotemporal dynamics of contractility-dependent adhesions. Nat Comm. 2015;6:8720. doi:10.1038/ncomms9720.

Friedl P, Bröcker EB. The biology of cell locomotion within three-dimensional extracellular matrix. Cell Mol Life Sci. 2000;57(2):41-64.

Friedl P, Gilmour D. Collective cell migration in morphogenesis, regeneration and cancer. Nat Rev Mol Cell Biol. 2009;10: 445.

Friedl P, Locker J, Sahai E, Segall JE. Classifying collective cancer cell invasion. Nat Cell Biol. 2012;14(8):777. 
Giri A, Bajpai S, Trenton N, Jayatilaka H, Longmore GD, Wirtz D. The arp2/3 complex mediates multigeneration dendritic protrusions for efficient 3-dimensional cancer cell migration. Faseb J. 2013;27:4089,

Gjorevski N, Piotrowski A, Varner VD, Nelson CM. Dynamic tensile forces drive collective cell migration through three-dimensional extracellular matrices. Sci Rep. 2015;5:11458. doi:10.1038/srep11458.

Gong X, Lin C, Cheng J, Su J, Zhao H, Liu T, Wen X, Zhao P. Generation of multicellular tumor spheroids with microwell-based agarose scaffolds for drug testing. PLoS ONE. 2015;10(6):0130348. doi:10.1371/journal.pone.0130348.

Grinnell F, Petroll WM. Cell motility and mechanics in three-dimensional collagen matrices. Annu Rev Cell Dev Biol. 2010;26:335-61.

Guo C, Kaufman LJ. Flow and magnetic field induced collagen alignment. Biomaterials. 2007;28(6):1105. doi:10.1016/j.biomaterials.2006.10.010.

Hahn MS, Miller JS, West JL. Three-dimensional biochemical and biomechanical patterning of hydrogels for guiding cell behavior. Adv Mater. 2006;18:2679.

Han C, Takayama S, Park J. Formation and manipulation of cell spheroids using a density adjusted peg/dex aqueous two phase system. Sci Reports. 2015;5(11891): doi:10.1038/srep11891.

Hirschhaeuser F, Menne H, Dittfeld C, West J, Mueller-Klieser W, Kunz-Schughart LA. Multicellular tumor spheroids: An underestimated tool is catching up again. J Biotech. 2010;148:3. doi:10.1016/j.jbiotec.2010.01.012.

Huang Y, Agrawal B, Sun D, Kuo JS, Williams JC. Microfluidics-based devices: New tools for studying cancer and cancer stem cell migration. Biomicrofluidics. 2011;5(1):013412. doi:10.1063/1.3555195.

Hunter KW, Crawford NP, Alsarraj J. Mechanisms of metastasis. Brest Cancer Res. 2008;10(Suppl 1):2.

Inch WR, McCredie JA, Sutherland RM. Growth of nodular carcinomas in rodents compared with multi-cell spheroids in tissue culture. Growth. 1970;34:271.

Junttila MR, de Sauvage FJ. Influence of tumour micro-environment heterogeneity on therapeutic response. Nature. 2013;501:346. doi:10.1038/nature12626.

Kim D, Provenzano PP, Smith CL, Levchenko A. Matrix nanotopography as a regulator of cell function. J Cell Biol. 2012;197(3):351. doi:10.1083/jcb.201108062.

Kim J, Jones CAR, Groves N, Sun B. Three-dimensional reflectance traction microscopy. PLoS ONE. 2016;11(6):0156797.

Kloxin AM, Kasko AM, Salinas CN, Anseth KS. Photodegradable hydrogels for dynamic tuning of physical and chemical properties. Science. 2009;324:59.

Lee J, Abdeen AA, Whcislo KL, Fan TM, Kilian KA. Interfacial geometry dictates cancer cell tumorigenicity. Nat Materials. 2016;15:865. doi:10.1038/nmat4610.

Liang L, Jones CAR, Chen S, Sun B, Jiao Y. Heterogeneous force network in 3d cellularized collagen networks. Phys Biol. 2016;13:066001.

Liu L, Doclos G, Sun B, Lee J, Wu A, Kam Y, Sontag E, Stone H, Sturm JC, Gatenby R, Austin RH. Minimization of thermodynamic costs in cancer cell invasion. Proc Nat Acad Sci. 2013;110(5):1686-91.

Liu L, Sun B, Pedersen J, Koh-Meng, Getzenberg RH, Stone HA, Austin RH. Probing the invasiveness of prostate cancer cells in a 3D microfabricated landscape. Proc Nat Acad Sci. 2011;108:6853-856.

Ma X, Schickel ME, Stevenson MD, Sarang-Sieminski AL, Gooch KJ, Ghadiali SN, Hart RT. Fibers in the extracellular matrix enable long-range stress transmission between cells. Biophys J. 2013;104:1410-18.

Mahmud G, Bishop CJCKJM, Komarova YA, Chaga O, Soh S, Huda S, Kandere-Grzybowska K, Grzybowski BA. Directing cell motions on micropatterned ratchets. Nat Phys. 2009;5:606. doi:10.1038/NPHYS1306.

Maruthamuthu V, Sabass B, Schwarz US, Gardel ML. Cell-ecm traction force modulates endogenous tension at cell-cell contacts. Proc Natl Acad Sci. 2011;108(12):4708.

McCredie JA, Sutherland RM, Inch WR. Growth of nodular carcinomas in rodents compared with multi-cell spheroids in tissue culture. J Natl Cancer Inst. 1971;46:113.

Nelson CM, Vanduijn MM, Inman JL, Fletcher DA, Bissell MJ. Tissue geometry determines sites of mammary branching morphogenesis in organotypic cultures. Science. 2006;314:298. doi:10.1126/science.1131000.

Notbohm J, Banerjee S, Utuje KJC, Gweon B, Jang H, Park Y, Shin J, Butler JP, Fredberg JJ, Marchetti MC. Cellular contraction and polarization drive collective cellular motion. Biophys J. 2016;110:2729.

Petrie RJ, Gavara N, chadwick RS, Yamada KM. Nonpolarized signaling reveals two distinct modes of $3 \mathrm{~d}$ cell migration. J Cell Biol. 2012;197(3):439.

Petrie RJ, Yamada KM. At the leading edge of three-dimensional cell migration. J Cell Sci. 2013;125:1-10.

Poujade M, Grasland-Mongrain E, Hertzog A, Jouanneau J, Chavrier P, Ladoux B, Buguin A, Silberzan P. Collective migration of an epithelial monolayer in response to a model wound. Proc Natl Acad Sci. 2007;104(41):15988-93.

Provenzano PP, Eliceiri KW, Campbell JM, Inman DR, White JG, Keely PJ. Collagen reorganization at the tumor-stromal interface facilitates local invasion. BMC Med. 2006;4(38):1221. doi:10.1186/1741-7015-4-38.

Rape AD, Guo WH, Wang YL. The regulation of traction force in relation to cell shape and focal adhesions. Biomaterials. 2011;32:2043.

Ravasio A, Cheddadi I, Chen T, Pereira T, Ong HT, Bertocchi C, Brugues A, Jacinto A, Kabla AJ, Toyama Y, Trepat X, Gov N, de Almeida LN, Ladoux B. Gap geometry dictates epithelial closure efficiency. Nat Comm. 2015;6(7683): doi:10.1038/ncomms8683.

Shin Y, Kim H, Han S, Won J, Lee E, Kamm RD, Kim J, Chung S. Extracellular matrix heterogeneity regulates three-dimensional morphologies of breast adenocarcinoma cell invasion. Adv Healthc Mater. 2014;2(6):790. doi:10.1002/adhm.201200320.

Singhvi R, Kumar A, Lopez GP, Stephanopoulos GN, Wang DI, Whitesides GM, Ingber DE. Engineering cell shape and function. Science. 1994;264(5159):696. doi:10.1126/science.8171320.

Sun B, Lembong J, Normand V, Rogers M, Stone HA. The spatial-temporal dynamics of collective chemosensing. Proc Nat Aca Sci. 2012;109(20):7759-764.

Theveneau E, Steventon B, Scarpa E, Garcia S, Trepat X, Streit A, Mayor R. Chase-and-run between adjacent cell populations promotes directional collective migration. Nat Cell Biol. 2013;15(7):763-72.

Timmins NE, Nielsen LK. Tissue Engineering. New York: Springer; 2007, p. 141. Chap. 10. 
Valencia AMJ, Wu P, Yogurtcu ON, Rao P, DiGiacomo J, Godet I, He L, Lee M, Gilkes D, Sun SX, Wirtz D. Collective cancer cell invasion induced by coordinated contractile stresses. Oncotarget. 2015;6(41):43438.

Varner VD, Nelson CM. Toward the directed self-assembly of engineered tissues. Ann Rev Chem Biomol Eng. 2014;5:507. doi:10.1146/annurev-chembioeng-060713-040016.

Villar G, Graham AD, Bayley H. A tissue-like printed material. Science. 2013;340:48. doi:10.1126/science.1229495.

Wang H, Abhilash AS, Chen CS, Wells RG, Shenoy VB. Long-range force transmission in fibrous matrices enabled by tension-driven alignment of fibers. Biophys J. 2014;107:2592.

Weiswald L, Bellet D, Dangles-Marie V. Spherical cancer models in tumor biology. Neoplasia. 2015;17(1):1. doi:10.1016/j.neo.2014.12.004

Yanagawa F, Sugiura S, Kanamori T. Hydrogel microfabrication technology toward three dimensional tissue engineering. Regen Ther. 2016;3:45. doi:10.1016/j.reth.2016.02.007.

Zimmermann J, Camley BA, Rappel W, Levine H. Contact inhibition of locomotion determines cell-cell and cell-substrate forces in tissues. Proc Natl Acad Sci. 2016;113(10):2660. doi:10.1073/pnas.1522330113.

Submit your manuscript to a SpringerOpen ${ }^{\circ}$ journal and benefit from:

- Convenient online submission

- Rigorous peer review

- Open access: articles freely available online

- High visibility within the field

- Retaining the copyright to your article

Submit your next manuscript at $\gg$ springeropen.com 